\title{
Opereration verbessert Überleben
}

Manche jüngere Brustkrebspatientinnen im Stadium IV profitieren möglicherweise von einer Operation als Ersttherapie, und zwar unabhängig von der Tumorgröße. Sie leben länger als Patientinnen ohne Operation, wie eine retrospektive Analyse von Daten des US-amerikanischen Registers SEER (Surveillance, Epidemiology, and End Results) vermuten lässt.

Für die abschließende Analyse standen die Befunde von 21.372 Patientinnen zur Verfügung, von denen 8.330 Frauen operiert worden waren. Dabei lag der Anteil der operierten Patientinnen 1988 noch bei knapp $68 \%$, im Jahr 2011 waren es nur noch etwa $25 \%$.

Das mediane Überleben lag bei Frauen, deren Karzinom zwischen 1988 und 1991 entdeckt worden war, bei 20 Monaten und war damit sechs Monate kürzer als bei Frauen mit der Krebsdiagnose zwischen 2007 und 2011. Auch wenn im Vergleich zum Studienbeginn im Verlauf immer seltener operiert wurde, lag die Wahrscheinlichkeit für ein längeres Überleben nach der Operation um $40 \%$ höher (Hazard Ratio 0,60; $p<0,001)$. Insgesamt betrachtet lebten Patientinnen ohne Operation als Erstmaßnahme median noch 19 Monate, mit Operation dagegen noch $28 \mathrm{Mo}-$ nate, und zwar unabhängig von der Tumorgröße. Betrug diese weniger als $2 \mathrm{~cm}$, lag die Differenz sogar bei elf Monaten (34 vs. 23 Monate). Am schlechtesten schnitten Patientinnen mit einem Tumordurchmesser über $5 \mathrm{~cm}$ ab.

Bei Patienten mit einer Krebsdiagnose vor 2002 war nach einer Operation die

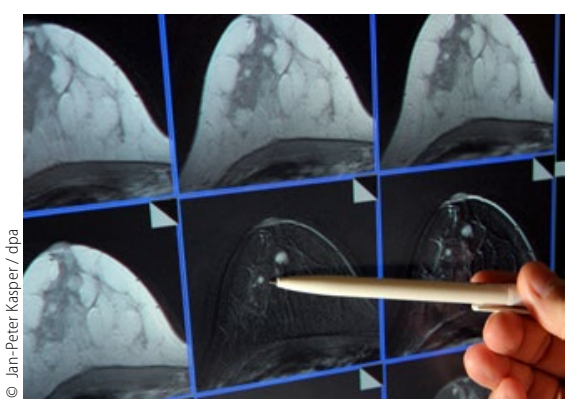

Entscheidende Frage bei Brustkrebs im Spätstadium: Operieren oder nicht?

Wahrscheinlichkeit des verlängerten Überlebens mehr als dreifach höher als ohne chirurgischen Eingriff (Odds Ratio [OR] 3,61). Nach einer Multivariatanalyse lag die OR immer noch bei 2,8 (p < 0,001).

Peter Leiner

Thomas A et al. Initial Surgery and Survival in Stage IV Breast Cancer in the United States, 1988-2011. JAMA Surg. 2015; Dec 2 [Epub ahead of print].

\section{Subgruppe profitiert von adjuvanter Antikörpertherapie}

$\mathrm{Zu}$ den derzeit untersuchten Strategien, um eine Trastuzumab-Resistenz zu überwinden, gehört die duale HER2-Blockade die gute Ergebnisse in der Erstlinientherapie bei HER2-positiven Patientinnen mit metastasiertem Mammakarzinom zeigten. Eine Metaanalyse randomisierter Studien untersuchte nun den möglichen Nutzen einer adjuvanten, gegen HER2 gerichteten Antikörpertherapie bei kleinen Läsionen bis zu $2 \mathrm{~cm}$ Durchmesser.

Insgesamt gingen Daten von 4.220 Patientinnen mit HER2-positiven Mammakarzinomen der Maximalgröße von $2 \mathrm{~cm}$ in die Analyse ein. Davon erhielten 2.588 eine adjuvante Therapie auf Trastuzumab-Basis. $94 \%$ der 2.263 Patientinnen mit Hormonrezeptor(HR)-positiven Tumoren hatten eine endokrine Therapie erhalten. Die kumulative Progressionsoder Rezidivinzidenz nach einer medianen Beobachtungsdauer von acht Jahren betrug für die Gruppe der HR-positiven, mit Trastuzumab behandelten Patientinnen $17,3 \%$ gegenüber $24,3 \%$ bei Patienmit neuen Agenzien plus Chemotherapie, tinnen ohne Antikörpertherapie. Dies entspricht einer Verbesserung im krankheitsfreien Überleben um $7 \%$. Die Auswertung zum Gesamtüberleben ergab für die Trastuzumab-Gruppe einen Vorteil von 3,8\%. Die kumulativen Inzidenzraten bezüglich des krankheitsfreien Über- lebens und des Gesamtüberlebens betrugen für hormonrezeptorpositive Patientinnen mit Nodalstatus N0 oder N1 $12,7 \%$ vs. $19,4 \%$. Die entsprechenden Ergebnisse für Patientinnen mit hormonrezeptornegativen Tumoren fielen generell ungünstiger aus. Wolfgang Zimmermann

O'Sullivan CC et al. Efficacy of adjuvant trastuzumab for patients with human epidermal growth factor receptor 2-positive early breast cancer and tumors $\leq 2 \mathrm{~cm}$ : a meta-analysis of the randomized trastuzumab trials. J Clin Oncol. 2015;33(24):2600-8.

\section{kurz notiert}

\section{MET als Achillessehne von Antitumortherapien}

Mutationen oder Amplifikationen des Protoonkogens MET sind an der Pathogenese verschiedener Tumoren beteiligt. MET wird nicht nur von Tumorzellen exprimiert, sondern auch von tumorassoziierten Stromazellen. Nun wurde nachgewiesen, dass MET für das Anlocken von Neutrophilen und die Zytotoxizität als Antwort auf den Hepatozytenwachstumsfaktor (HGF) verantwortlich ist. Eine METDeletion in Neutrophilen von Mäusen verstärkt Tumorwachstum und Metastasierung. Darüber hinaus ist MET für die Absonderung von Neutrophilen bei einer Kolitis, Rash oder Peritonitis notwendig. MET wird durch den Tumornekrosefaktor alpha oder andere inflammatorische Stimuli in Neutrophilen induziert. Eine HGF/MET-abhängige Stickoxid-Freisetzung durch Neutrophile fördert das Absterben von Krebszellen.

Der therapeutische Nutzen eines MET-Kinase-Inhibitors wird von dem protumoralen Effekt der METBlockade in den Neutrophilen teilweise gehemmt. Dementsprechend scheint MET in den Neutrophilen die Achillessehne gegen MET gerichteter Krebstherapien zu sein [Finisguerra V et al. Nature. 2015;522(7556):349-53]. Judith Neumaier 